\title{
Evaluación de los componentes del manejo antes, durante y después de la matanza y su asociación con la presencia de carne DFD en bovinos del noreste de México
}

Jorge Loredo Osti ${ }^{\text {a,b }}$

Eduardo Sánchez López ${ }^{\mathrm{a} *}$

Alberto Barreras Serrano ${ }^{a}$

Fernando Figueroa Saavedra ${ }^{\text {a }}$

Cristina Pérez Linares ${ }^{a}$

Miguel Ruiz Albarrán b

a Universidad Autónoma de Baja California. Instituto de Investigaciones en Ciencias Veterinarias. Laguna Campestre, Mexicali. BC. México.

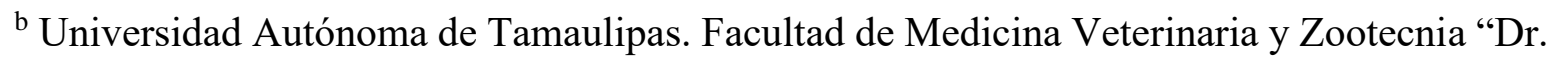
Norberto Treviño Zapata". Tamaulipas, México.

* Autor de correspondencia: edsanmxl@hotmail.com

\section{Resumen:}

Un total de 27 variables de manejo (antes, durante y después del sacrificio) en 394 bovinos fueron analizadas y utilizadas para determinar su asociación y valor explicativo con la presencia de carne DFD (Dark, Firm, Dry, por sus siglas en inglés), mediante las razones de probabilidad en un modelo de regresión logística múltiple. El estudio se realizó de noviembre de 2016 a agosto de 2017 en un rastro Tipo Inspección Federal localizado en el noreste de México. La presencia de carne DFD fue del $13.45 \%$. Se realizó un contraste entre clases para los factores evaluados mediante t Student y Ji cuadrada en función de la naturaleza de la variable como criterio de inclusión en la modelación logística. Diez de las variables 
mostraron significación estadística $(P<0.05)$ en estas pruebas, pero solo cuatro de ellas presentaron valor explicativo en el modelo logístico múltiple final $(P<0.01)$, las cuales fueron: el tiempo de espera previo a la muerte, un mal insensibilizado, el espesor de la grasa subcutánea y diferencial de $\mathrm{pH}$ de la canal establecido con $24 \mathrm{~h}$ de diferencia. Las dos primeras aumentaron la posibilidad en la presencia de carne DFD, por el contrario, el espesor de grasa y el diferencial de $\mathrm{pH}$ fueron inversamente proporcionales. Las cuatro variables incluidas en el modelo final estuvieron presentes en diferentes etapas y son de naturaleza distinta. Por esta razón, para prevenir de manera efectiva este problema se necesita una evaluación multicausal en todo el proceso de matanza.

Palabras clave: Carne, Corte oscuro, DFD, Bovino.

Recibido: $26 / 04 / 2018$

Aceptado: 29/11/2019

\section{Introducción}

La carne DFD (Dark, Firm, Dry, por sus siglas en inglés) es un problema que afecta la calidad sanitaria, fisicoquímica y sensorial del producto ${ }^{(1,2)}$, debido a un $\mathrm{pH}$ final alto $(>5.8)$ que favorece el crecimiento de flora bacteriana y disminuye la vida de anaquel ${ }^{(3,4)}$. Además, la carne DFD presenta un color rojo oscuro y textura pegajosa, que da la apariencia de ser carne de animales viejos o que ha sido almacenada durante mucho tiempo ${ }^{(5,6)}$. Esto propicia una baja aceptación por el consumidor y origina pérdidas económicas a los productores ${ }^{(7,8)}$. En México, se calcula que la pérdida por cada canal con características DFD es de 88.58 dólares estadounidenses $^{(9)}$, superior a los 5.43 dólares de pérdida por canal encontrada en EE.UU ${ }^{(10)}$. La carne DFD presenta las siguientes características: incremento en la capacidad de retención de agua, palatabilidad pobre, menor terneza y mayor absorción de luz, lo cual afecta su aptitud tecnológica para la elaboración de varios productos cárnicos ${ }^{(11,12,13)}$.

La velocidad en la disminución del $\mathrm{pH}$ post mortem, está directamente relacionada con el nivel de estrés que sufre el animal antes de la matanza ${ }^{(14,15)}$. El estrés crónico y la exposición de largos plazos de estrés agudo, justo antes de ser sacrificado, hacen que las reservas de glucógeno muscular se consuman rápidamente ${ }^{(2)}$, reduciendo la cantidad de ácido láctico que se forma por la glucólisis anaerobia en el músculo luego de la muerte del animal, lo cual provoca la presencia de carne DFD, también llamada Corte Oscuro ${ }^{(16,17)}$. Existen diferentes factores intrínsecos que aumentan el riesgo de una mayor presencia de carne DFD tales como: 
el origen racial (más frecuente en Bos indicus) ${ }^{(18,19)}$, el sexo (mayor en machos enteros) $)^{(7,20)}$, el peso (menos común en ganado de mayor peso vivo) ${ }^{(21,22)}$, la cantidad de grasa (se presenta más frecuentemente con valores bajos en el espesor de la grasa subcutánea) ${ }^{(4,23)}$ y la edad (es más frecuente en animales viejos) ${ }^{(24,25)}$. Las condiciones ambientales también influyen en la presencia de carne DFD, las temperaturas extremas provocan estrés por calor o por frío ${ }^{(26,27)}$.

El manejo inadecuado en el procesamiento ante mortem es uno de los principales factores desencadenantes del corte oscuro, las distancias largas en el transporte y la elevada densidad animal en espacios reducidos influyen en su presencia, así como los tiempos prolongados en los corrales de espera, en las mangas y en el cajón de sacrificio ${ }^{(15,20)}$, el uso de instrumentos estresantes (arreadores eléctricos, reatas, palos, etc.) en el arreo hacia el cajón de insensibilizado y una mala efectividad de este último también se han reportado como factores de riesgo ${ }^{(28,29)}$. El proceso post sacrificio también repercute en la presencia de carne DFD, la tasa de disminución del pH y la temperatura del músculo interactúan continuamente durante el rigor mortis, y son probablemente dos de los factores post mortem más importantes que afectan las propiedades de la carne, tales como: color, $\mathrm{pH}$ final, capacidad de retención de agua y terneza ${ }^{(30,31,32)}$, algunas características de la canal como su peso y el espesor de grasa subcutánea influyen en esta interacción ${ }^{(33,34)}$.

Estudios como los anteriores se han realizado para establecer la asociación entre la presencia de carne DFD y los factores evaluados; sin embargo, la mayoría de ellos han establecido esta asociación analizando dichos factores de manera individual y aislada, lo cual no permite analizar el efecto de las variables de manera conjunta ni sus interacciones. Por lo anterior, el objetivo de este trabajo fue evaluar la asociación de factores con valor explicativo, así como sus interacciones, relacionados con el manejo antes, durante y después del sacrificio, sobre la presencia de carne DFD.

\section{Material y métodos}

Se evaluaron 27 variables intrínsecas y extrínsecas antes, durante y después del sacrificio, en las cuatro estaciones del año. El trabajo se efectuó entre noviembre de 2016 y agosto de 2017 en una planta de sacrificio Tipo Inspección Federal (TIF) localizada en Cd. Victoria, Tamaulipas. Los registros de las variables se tomaron en 16 períodos de 3 días cada uno: a la llegada, durante el sacrifico y el procesamiento de la canal. El genotipo de los animales correspondió, principalmente, a cruces comerciales de Bos taurus x Bos indicus. Los bovinos arribaron desde distintos puntos de la región y en diferente tipo de vehículo. 


\section{Tamaño de muestra}

El número de animales se determinó para un muestreo simple aleatorio por atributos, considerando una población finita ${ }^{(35)}$. Los componentes de la fórmula fueron un valor de confianza del $95 \%(Z=1.96)$, precisión del 5\%, un estimador de varianza igual a 0.25 [ $\sigma^{2}=$ $\pi(1-\pi)$ ] y un valor de $\mathrm{N}$ generado del centro de sacrificio de los últimos tres años $(\mathrm{n}=38,950$ animal/año). El tamaño de muestra obtenido $(n=394)$ fue distribuido proporcionalmente por el número de sacrificio en las estaciones del año: primavera $=95$, verano $=110$, otoño $=78 \mathrm{e}$ invierno $=110$. La toma de datos se realizó en los meses de noviembre de 2016, febrero, mayo y agosto de 2017.

\section{Recopilación de información}

\section{Variables ante mortem}

Al arribo al matadero se registraron las variables intrínsecas y las extrínsecas tales como prácticas de transporte, forma de adquisición del animal, estación del año, temperatura y humedad relativa (termohigrómetro con una sonda, Hanna instruments, modelo HI9565). En los corrales de descanso se registró la presencia de lesiones visibles y variables concernientes al espacio y tiempo de permanencia. También se registró la separación de algún animal en corrales individuales por motivos de comportamiento o salud (arisco, montas o lesiones). Antes de la matanza se registró el día de la semana y los datos individuales referentes al tiempo de permanencia en la manga que conduce a los animales al cajón de insensibilización y a las condiciones del arreo del ganado. Se midió la temperatura y la humedad relativa antes de entrar en el cajón de sacrificio. Se obtuvo el índice de temperatura-humedad relativa (ITHR) mediante la fórmula siguiente: ITH $=[0.81 * T]+\mathrm{HR} / 100 *(\mathrm{~T}-14.4)+46.4$, donde $\mathrm{T}=$ Temperatura ambiente $\left({ }^{\circ} \mathrm{C}\right)$ y $\mathrm{HR}=$ Humedad relativa $(\%)^{(36)}$.

\section{Variables durante la matanza}

El aturdimiento se realizó mediante una pistola de perno cautivo. Durante el colgado del cuerpo del animal se valoró la eficacia de la insensibilización mediante el registro de los indicadores de comportamiento siguientes: parpadeo espontáneo, rotación total de globo ocular, respiración rítmica, intento de levantarse, enderezamiento y vocalizaciones. Se 
consideró una incorrecta insensibilización del animal cuando éste presentaba cualquiera de los signos anteriores. También se determinó el intervalo aturdimiento-desangrado desde que el animal colapsó hasta el degüello ${ }^{(37,38)}$.

\section{Variables en la canal caliente}

Se registró el peso de la canal caliente; además, 45 min después del sacrificio se registró (por triplicado) el valor del $\mathrm{pH}\left(\mathrm{pH}_{45 \mathrm{~min}}\right)$ con el fin de establecer un diferencial $(\Delta \mathrm{pH})$ entre el $\mathrm{pH}_{45 \min } \mathrm{y}$ el $\mathrm{pH}$ último ( $\mathrm{pHu}$ ) valorado $24 \mathrm{~h}$ después, el $\mathrm{pH}$ fue medido con un potenciómetro que contaba con un dispositivo de punción para carne (Hanna instruments, modelo HI99163). También se registró la temperatura (por triplicado) de la canal a los $45 \mathrm{~min}$, en el músculo Longissimus dorsi a $5 \mathrm{~cm}$ de penetración (termómetro con sonda de penetración, Hanna instruments, modelo HI935007N).

\section{Variables en la canal fría}

En el cuarto frío $\left(2{ }^{\circ} \mathrm{C}\right) 24 \mathrm{~h}$ después de la matanza se registraron los valores del $\mathrm{pH}$ final, el espesor de la grasa subcutánea y los parámetros colorimétricos: $\mathrm{L}^{*}=\operatorname{Luminosidad~(0~a~100),~}$ $\mathrm{a}^{*}=$ índice de rojo (-60 a 60) y b* = índice de amarillo (-60 a 60). Todos estos registros se realizaron por triplicado en el área del músculo Longissimus dorsi entre la $10{ }^{a}$ y $12 .{ }^{a}$ costilla de la media canal izquierda, 30 min después de haber realizado el corte. El espesor de la grasa subcutánea se determinó con un calibrador Vernier de acero inoxidable y los valores de color con un espectrofotómetro Minolta con $5 \mathrm{~cm}$ de apertura, iluminante $\mathrm{C}$ y observador de $2^{\circ}$ (Modelo CR-410, Minolta Co., Ltd., Osaka, Japón,). El Chroma (0 a 200) se calculó utilizando la siguiente ecuación: $\mathrm{C}^{*}=\left(\mathrm{a}^{* 2}+\mathrm{b}^{* 2}\right)^{1 / 2(39)}$. Por último, se registró la densidad en la cámara fría (número de canales $/ \mathrm{m}^{2}$ ).

\section{Variables de clasificación}

Según los criterios establecidos, la canal se clasificó en oscura, firme y no exudativa (DFD) con base en lo siguiente: $\mathrm{pHu} \geq 5.8, \mathrm{~L}^{*}<40$ y $\mathrm{C}^{*}<30^{(40)}$. Las canales que presentaron criterios diferentes se clasificaron como normales. 


\section{Análisis de las variables}

El contraste entre las clases DFD y normal para las variables estudiadas se realizó en función de la naturaleza de la variable: Se utilizó t de Student para las variables cuantitativas continuas, mientras Ji-cuadrada y exacta de Fisher (para frecuencia $<5$ en una casilla) se utilizaron para las variables categóricas. La significancia fue establecida cuando $P<0.05$.

\section{Estudio de asociación}

La asociación de los factores de estudio con la clasificación de la carne (variable dependiente) de naturaleza binomial $(1=$ DFD, $0=$ normal), se realizó aplicando un modelo logístico con múltiples variables independientes, así como sus interacciones. Como primer paso en la utilización del modelo logístico, se incluyeron las variables con significancia estadística $(P<0.05)$ en la comparación entre clases DFD y normal. Del modelo completo se excluyeron los factores que resultaron no significativos $(P \geq 0.05)$ según la prueba de Wald. Esto permitió obtener el modelo final con sus razones de probabilidad (OR, por sus siglas en inglés), errores estándar (EE) e intervalos de confianza (IC 95\%). Al modelo final se le realizó la prueba de bondad de ajuste de Hosmer-Lemeshow ${ }^{(41,42)}$. Los contrastes entre clases DFD y normal para las variables estudiadas, así como el análisis del modelo logístico con múltiples variables independientes se realizaron al aplicar los procedimientos TTEST, FREQ y LOGISTIC del paquete estadístico SAS $9.4^{(43)}$.

\section{Resultados y discusión}

El porcentaje de carne DFD encontrada en este estudio fue de $13.45 \%$, inferior al $38.99 \%$ observado en el último estudio realizado en otra región de México ${ }^{(41)}$. Las diferencias regionales entre la presencia de carne DFD sugieren que los factores que influyen en esta condición son múltiples y variados ${ }^{(44)}$. Además, se ha observado un aumento de la frecuencia de este problema en otros países de Norteamérica: En EE.UU. pasó de $1.9 \%$ en $2005^{(45)}$ a $3.2 \%$ en $2012^{(46)}$ y en Canadá de $1.0 \%$ a $1.3 \%$ en un lapso de poco más de una década ${ }^{(47,48)}$.

De las variables incluidas en este estudio, solo 10 mostraron significancia en el contraste DFD vs normal (Cuadros 1 y 2). Sin embargo, al realizar el análisis del modelo de regresión logística múltiple, únicamente cuatro de ellas mostraron valor explicativo $(P<0.05)$, las cuales fueron: tiempo en corral de espera, eficacia del aturdimiento, espesor de grasa 
subcutánea (EGS) y el diferencial de $\mathrm{pH}(\Delta \mathrm{pH})$ (Cuadro 3). Ninguna de las interacciones entre estas variables o con las otras restantes mostraron valor significativo dentro del modelo $(P>0.05)$. En la prueba de bondad de ajuste de Hosmer-Lemeshow, no se rechazó la hipótesis nula $(P=0.963)$. En el Cuadro 4 se presentan los valores de OR, junto con su intervalo de confianza al $95 \%$.

Cuadro 1: Efecto de variables cuantitativas, intrínsecas y extrínsecas, sobre el tipo de carne de bovino

\begin{tabular}{|c|c|c|c|c|c|}
\hline \multirow[t]{2}{*}{ Variable } & \multicolumn{2}{|l|}{ DFD } & \multicolumn{3}{|c|}{ Normal } \\
\hline & Media & $\mathbf{E E}$ & Media & $\mathbf{E E}$ & Valor $P$ \\
\hline \multicolumn{6}{|l|}{ Desembarque } \\
\hline Temperatura, ${ }^{\circ} \mathrm{C}$ & 30.4 & 1.00 & 32.5 & 0.28 & 0.010 \\
\hline Índice HR-T & 77.1 & 10.5 & 79.7 & 0.28 & 0.934 \\
\hline \multicolumn{6}{|l|}{ Transporte } \\
\hline Densidad animal, $\mathrm{m}^{2} /$ cabeza & 3.1 & 0.29 & 2.6 & 0.09 & 0.061 \\
\hline \multicolumn{6}{|l|}{ Corral de descanso } \\
\hline Densidad animal, $\mathrm{m}^{2} /$ cabeza & 9.6 & 1.17 & 11.5 & 0.55 & 0.214 \\
\hline Tiempo, h & 15.4 & 0.23 & 14.8 & 0.10 & 0.021 \\
\hline \multicolumn{6}{|c|}{ Manga de conducción al cajón de matanza } \\
\hline $\mathrm{N}^{\circ}$ de personas en arreo & 1.5 & 0.13 & 1.7 & 0.07 & 0.449 \\
\hline Temperatura, ${ }^{\circ} \mathrm{C}$ & 23.6 & 0.80 & 25.4 & 0.24 & 0.009 \\
\hline Índice HR-T & 71.2 & 1.12 & 74.1 & 0.33 & 0.002 \\
\hline Tiempo, min & 68.2 & 6.47 & 54.4 & 2.18 & 0.023 \\
\hline \multicolumn{6}{|l|}{ Matanza } \\
\hline Intervalo aturdimiento-desangrado, seg & 179.8 & 10.04 & 147.7 & 4.49 & 0.008 \\
\hline \multicolumn{6}{|l|}{ Canal caliente } \\
\hline Peso, $\mathrm{kg}$ & 284.7 & 10.32 & 295.8 & 3.78 & 0.289 \\
\hline $\mathrm{pH}_{45 \min }$ & 7.0 & 0.03 & 6.9 & 0.02 & 0.131 \\
\hline Temperatura, ${ }^{\circ} \mathrm{C}_{45 \min }$ & 33.2 & 0.31 & 33.5 & 0.11 & 0.351 \\
\hline \multicolumn{6}{|l|}{ Canal fría } \\
\hline$\Delta \mathrm{pH}$ & 0.95 & 0.04 & 1.45 & 0.02 & $<0.001$ \\
\hline Espesor de grasa, $\mathrm{cm}$ & 0.40 & 0.04 & 0.55 & 0.02 & 0.007 \\
\hline Densidad en el cuarto frío, $\mathrm{m}^{2} /$ canal & 2.3 & 0.06 & 2.2 & 0.03 & 0.667 \\
\hline
\end{tabular}


Cuadro 2: Efecto de variables categóricas, intrínsecas y extrínsecas, sobre el tipo de carne de bovino

\begin{tabular}{|c|c|c|c|c|c|}
\hline \multirow[t]{2}{*}{ Variable } & \multicolumn{2}{|l|}{ DFD } & \multicolumn{3}{|l|}{ Normal } \\
\hline & Frecuencia & Porcentaje & Frecuencia & Porcentaje & Valor $P$ \\
\hline \multicolumn{6}{|l|}{ Animal } \\
\hline Sexo & & & & & $0.186^{(1)}$ \\
\hline Macho & 11 & 20.00 & 44 & 80.00 & \\
\hline Hembra & 42 & 12.39 & 297 & 87.61 & \\
\hline \multicolumn{6}{|l|}{ Origen } \\
\hline \multicolumn{3}{|c|}{ Forma de adquisición } & & & $0.895^{(1)}$ \\
\hline Subasta & 18 & 14.17 & 109 & 88.89 & \\
\hline Granja & 35 & 13.11 & 232 & 86.49 & \\
\hline \multicolumn{6}{|l|}{ Desembarque } \\
\hline Época & & & & & $0.097^{(1)}$ \\
\hline Primavera & 6 & 6.32 & 89 & 93.68 & \\
\hline Verano & 15 & 13.51 & 96 & 86.49 & \\
\hline Otoño & 14 & 17.95 & 64 & 82.05 & \\
\hline Invierno & 18 & 16.36 & 92 & 83.64 & \\
\hline \multicolumn{6}{|l|}{ Transporte } \\
\hline Distancia & & & & & $0.048^{(1)}$ \\
\hline$>60 \mathrm{~min}$ & 9 & 25.71 & 26 & 74.29 & \\
\hline $30-60 \mathrm{~min}$ & 6 & 8.45 & 65 & 91.55 & \\
\hline$<30 \mathrm{~min}$ & 38 & 13.19 & 250 & 86.81 & \\
\hline Tipo de transpor & & & & & $0.191^{(1)}$ \\
\hline$<2 \mathrm{~m}$ largo & 24 & 13.87 & 149 & 86.13 & \\
\hline 2-4 m largo & 6 & 25.00 & 18 & 75.00 & \\
\hline$>4 \mathrm{~m}$ largo & 23 & 11.68 & 174 & 88.32 & \\
\hline \multicolumn{6}{|c|}{ Corrales de descanso } \\
\hline Separación & & & & & $0.741^{(2)}$ \\
\hline $\mathrm{Si}$ & 3 & 15.00 & 17 & 85.00 & \\
\hline No & 50 & 13.37 & 324 & 86.63 & \\
\hline Lesiones visible & & & & & $0.293^{(2)}$ \\
\hline $\mathrm{Si}$ & 2 & 25.00 & 6 & 75.00 & \\
\hline No & 51 & 13.21 & 335 & 86.79 & \\
\hline \multicolumn{6}{|c|}{ Manga de conducción al cajón de matanza } \\
\hline \multicolumn{3}{|c|}{ Instrumento de arreo } & & \multicolumn{2}{|c|}{$0.070^{(1)}$} \\
\hline Picana & 25 & 15.92 & 132 & 84.08 & \\
\hline Otro & 6 & 6.38 & 88 & 93.62 & \\
\hline Ninguno & 22 & 15.38 & 121 & 84.62 & \\
\hline Caídas & & & & & $89^{(2)}$ \\
\hline
\end{tabular}




\begin{tabular}{|c|c|c|c|c|c|}
\hline $\mathrm{Si}$ & 2 & 50.00 & 2 & 50.00 & \\
\hline No & 51 & 13.08 & 339 & 86.92 & \\
\hline \multicolumn{6}{|l|}{ Matanza } \\
\hline \multicolumn{5}{|c|}{ Día de la semana } & $0.771^{(1)}$ \\
\hline Lunes & 30 & 14.15 & 182 & 85.85 & \\
\hline Jueves & 23 & 12.64 & 159 & 87.36 & \\
\hline \multicolumn{5}{|c|}{ Eficacia en aturdimiento } & $<0.001^{(1)}$ \\
\hline Correcto & 25 & 9.29 & 244 & 90.71 & \\
\hline Incorrecto & 28 & 22.40 & 97 & 77.60 & \\
\hline
\end{tabular}

Valor $P$ de la prueba $\chi^{2}$ (1) y exacta de Fisher (2).

Cuadro 3: Coeficiente, error estándar y valor de p de las variables incluidas en el modelo logístico múltiple

\begin{tabular}{llll}
\hline Variable & Coeficiente & Error estándar & Valor de $\boldsymbol{P}$ \\
\hline Tiempo en corral, h & 0.522 & 0.126 & $<0.0001$ \\
Eficacia de aturdimiento & 1.251 & 0.375 & 0.0009 \\
Espesor de grasa subcutánea, cm & -1.883 & 0.636 & 0.0031 \\
Diferencial de $\mathrm{pH}[\Delta \mathrm{pH}]$ & -4.554 & 0.695 & $<0.0001$ \\
Constante & -5.308 & & \\
\hline
\end{tabular}

Cuadro 4: Razón de probabilidad (OR) e intervalo de confianza (IC) de las variables incluidas en el modelo logístico

\begin{tabular}{lll}
\hline Variable & OR & IC 95\% \\
\hline Tiempo en corral, h & 1.686 & 1.317 a 2.159 \\
Aturdimiento incorrecto & 3.492 & 1.674 a 7.287 \\
Espesor de grasa subcutánea,cm & 0.152 & 0.044 a 0.529 \\
Diferencial de $\mathrm{pH}[\Delta \mathrm{pH}]$ & 0.011 & 0.003 a 0.041 \\
\hline
\end{tabular}

El tiempo previo al sacrificio que el ganado pasa en los corrales de espera está asociado con la presencia de carne DFD, el valor de OR indica que la posibilidad de que se presente este defecto en las canales es 1.69 veces más grande por cada hora que transcurra. Algunos autores recomiendan un tiempo de reposo de $3 \mathrm{~h}$ como suficiente para que el animal se recupere de los efectos negativos derivados del transporte ${ }^{(15,49)}$, sin embargo, la normatividad de México y de otros países indica que el tiempo de descanso de los animales en el matadero debe ser de 12 a $24 \mathrm{~h}^{(50,51)}$, considerando el valor de OR obtenido en este estudio, la aplicación del tiempo máximo de estas normas implica un incremento importante en el riesgo de presencia de carne DFD. Tiempos de espera mayores de 15.8 h y de $12.0 \mathrm{~h}$ en corrales de retención, evaluados en dos estudios diferentes, han resultado en valores de OR de 2.20 y 2.03 respectivamente, estimados al aplicar modelos de regresión logística para canales con $\mathrm{pH}$ 
final $\geq 5.8^{(7,52)}$. Los resultados de este trabajo, así como los referidos anteriormente demuestran que a mayor tiempo que el animal pasa en el corral de descanso, más elementos estresantes pueden presentarse, aumentando la posibilidad de mayor frecuencia de carne DFD.

Una mala insensibilización del animal en este rastro mostró una posibilidad de 3.49 veces mayor de resultar en carne de tipo corte oscuro; por esto, en la matanza del ganado es importante determinar si el animal está insensible luego del disparo, ya que el desangrado y el procesamiento de la canal no pueden comenzar sin haberse realizado de manera correcta esta etapa ${ }^{(16,53)}$. Para que la eficacia de la insensibilización en el izado sea reconocida como "aceptable", debe presentarse un porcentaje no mayor al $0.2 \%$ de animales con signos de sensibilidad $^{(29)}$. En este estudio, el porcentaje de animales con signos de sensibilidad en el izado fue de $31.7 \%$, lo cual indica que, además de afectar negativamente la calidad de la carne, existe un problema serio de bienestar animal; esta problemática no es exclusiva del rastro evaluado, ya que el porcentaje encontrado fue menor al $49.0 \%$ reportado en otro rastro TIF del noroeste de México ${ }^{(54)}$ y al $66.9 \%$ de otro centro de matanza en Chile ${ }^{(15)}$. En relación al número de disparos se observaron los siguientes porcentajes: 1 (88.1\%), 2 (9.6\%) y 3 o más $(2.3 \%)$. Se considera como "aceptable" cuando el porcentaje de animales aturdidos instantáneamente con un solo disparo es del $95 \%$ o más, y como "problema grave" cuando no alcanza el $90 \%{ }^{(29)}$; en este centro de matanza no se llegó a esta última cifra, evidenciando la problemática de bienestar animal en esta etapa. Las causas más frecuentes de la baja eficacia en la insensibilización mediante el disparo con perno retráctil, son el mantenimiento inadecuado de la pistola o la fatiga que el operario experimenta debido a una alta velocidad del flujo de animales en el cajón de aturdimiento ${ }^{(55)}$. Aunque existen estudios que han examinado el impacto de un mal insensibilizado sobre la presencia de carne DFD $^{(28)}$, la mayoría de las investigaciones sobre el insensibilizado en bovinos han puesto mayor atención en las reacciones conductuales y fisiológicas relativas al bienestar animal ${ }^{(29,56)}$. Sin embargo, debe evaluarse de manera más exhaustiva la eficiencia del aturdimiento sobre la calidad de la canal ${ }^{(57)}$, ya que el insensibilizado es parte muy importante del proceso de matanza y, por lo tanto, puede afectar la calidad del producto final ${ }^{(58)}$.

El EGS presentó una relación inversamente proporcional sobre la presencia de carne DFD. El valor de OR de 0.15 indica que es un factor protector. Su inverso indica que por cada $\mathrm{cm}$ de aumento en EGS, existe una posibilidad de 6.67 veces mayor de resultar carne normal. Este resultado fue similar al obtenido en otra investigación que aplicó el sistema de clasificación de grado de engrasamiento de canal SEUROP, donde se observó un OR de 0.18 para canales con buen grado de engrasamiento ${ }^{(7)}$. Se estima que canales con un EGS menor a $0.76 \mathrm{~cm}$ tienen una mayor probabilidad de presentar carne $\mathrm{DFD}^{(4)}$. Canales con mayor engrasamiento mantienen una temperatura similar al animal vivo por más tiempo cuando son introducidas al cuarto frio ${ }^{(23)}$, acelerando el metabolismo muscular y presentando una mayor caída de $\mathrm{pH}$ en el proceso de instauración del rigor mortis ${ }^{(30)}$. 
La velocidad de disminución del $\mathrm{pH}$ en el músculo post-rigor tiene influencia directa sobre el pHu y el color de las canales. La relación observada entre el $\Delta \mathrm{pH}$ y la presencia de carne DFD resultó inversamente proporcional, con un valor de 0.011 para el OR. Su inverso indica que, por cada incremento en una unidad, la posibilidad de que se presente carne normal será 90.9 veces más grande. Existe una relación directa entre la velocidad de descenso del pH y la temperatura de la canal ${ }^{(32,59)}$. Canales con temperaturas más altas en el período pre-rigor generan valores de $\Delta \mathrm{pH}$ más altos, por lo tanto, con menor posibilidad de resultar en corte oscuro $^{(30)}$.

\section{Conclusiones e implicaciones}

El porcentaje en la presencia de carne DFD obtenido en este estudio fue de $13.45 \%$. De las 27 variables evaluadas, 10 de ellas, intrínsecas y extrínsecas, revelaron asociación estadística con la presencia de carne DFD, sin embargo, solo cuatro de estas diez mostraron valor explicativo para cuantificar el riesgo a corte oscuro dentro del modelo matemático utilizado; estas fueron: tiempo en corral de espera, eficacia del insensibilizado (donde se observaron problemas de bienestar animal), $\Delta \mathrm{pH}$ y EGS. Las tres primeras están presentes en todo el proceso de matanza; desde el manejo que se da a los animales antes y durante la muerte, así como en el metabolismo post mortem, la última es propia del animal. Por lo anterior, es necesaria una evaluación multicausal en todo el proceso de matanza para prevenir de manera adecuada este problema. En general, este estudio presenta datos concretos sobre qué factores realmente favorecen la presencia de carne DFD, con un interés directo para la planta de sacrificio en sí y para aquellas que trabajan en condiciones similares (TIF), pero también para fines científicos.

\section{Literatura citada:}

1. Alende M, Volpi-Lagreca G, Pordomingo AJ, Pighín D, Grigioni G, Carduza F, et al. Efectos del tiempo de transporte, espera pre-faena y maduración en novillos sobre indicadores de estrés, calidad instrumental y sensorial de la carne. Arch Med Vet 2014;(46):217-227.

2. Adzitey F, Nurul H. Pale soft exudative (PSE) and dark firm dry (DFD) meats: causes and measures to reduce these incidences - a mini review. Int Food Res J 2011;(18):1120.

3. Lawrie RA, Ledward DA. Lawrie's meat science. 7th ed. Abington Hall, England. Published by Woodhead Publishing Limited; 2006. 
4. Page JK, Wulf DM, Schwotzer TR. A survey of beef muscle color and pH. J Anim Sci 2001;79(3):678-687.

5. Hughes J, Clarke F, Purslow P, Warnerd R. High $\mathrm{pH}$ in beef Longissimus thoracis reduces muscle fibre transverse shrinkage and light scattering which contributes to the dark colour. Food Res Int 2017;(101):228-238.

6. Sawyer J, Apple J, Johnson Z, Baublits R, Yancey J. Fresh and cooked color of dark cutting beef can be altered by post-rigor enhancement with lactic acid. Meat Sci 2009;83(2):263-270.

7. Mach N, Bach A, Velarde A, Devant M. Association between animal, transportation, slaughterhouse practices, and meat pH in beef. Meat Sci 2008;(78):232-238.

8. Viljoen HF, de Kock HL, Webb EC. Consumer acceptability of dark, firm and dry (DFD) and normal pH beef steaks. Meat Sci 2001;(61):181-185.

9. Leyva-García IA, Figueroa-Saavedra F, Sánchez-López E, Pérez-Linares C, BarrerasSerrano A. Economic impact of DFD beef in a Federal Inspection Type (TIF) slaughterhouse. Arch Med Vet 2012;(44):39-42.

10. Miller M. Dark firm and dry beef. Beef facts product enhancement. Texas Tech University. 2007 https://fyi.extension.wisc.edu/wbic/files/2011/04/Dark-Firm-andDry-Beef.pdf. Accessed Aug 20, 2019.

11. Apple JK, Kegley EB, Galloway DL, Wistuba TJ, Rakes LK. Duration of restraint and isolation stress as a model to study the dark-cutting condition in cattle. J Anim Sci 2005;(83):1202-1214.

12. Zhang SX, Farouk MM, Young OA, Wieliezko KJ, Podmore C. Functional stability of frozen normal and high pH beef. Meat Sci 2005;(69):765-772.

13. Huff-Lonergan E, Lonergan SM. Mechanisms of water-holding capacity of meat: The role of postmortem biochemical and structural changes. Meat Sci 2005;(71):194-204,

14. Ferguson DM, Warner RD. Have we underestimated the impact of pre-slaughter stress on meat quality in ruminants? Meat Sci 2008;(80):12-19.

15. Gallo C, Teuber M, Cartes M, Uribe H, Grandin T. Improvements in stunning of cattle with a pneumatic stunner after changes in equipment and employee training. Arch Med Vet 2003;(35):159-170.

16. Chambers PG, Grandin T. Guidelines for humane handling, transport and slaughter of livestock. FAO-HIS. 2001. 
17. Mota D, Huertas SM, Alarcón AD, Pérez C, Guerrero I, Carrasco A, et al. Músculo oscuro, firme y seco: mecanismos involucrados. En: Mota D, et al, editores. Bienestar animal. Una visión global en Iberoamérica. 3a ed. Barcelona, España: Elsevier; 2016: 447-493.

18. Curley KO, Paschal JC, Welsh TH, Randel RD. Technical note: Exit velocity as a measure of cattle temperament is repeatable and associated with serum concentration of cortisol in Brahman bulls. J Anim Sci 2006;(84):3100-3103.

19. King DA, Schuehle CE, Randel RD, Welsh TH, Oliphint RA, Baird BE, et al. Influence of animal temperament and stress responsiveness on the carcass quality and beef tenderness of feedlot cattle. Meat Sci 2006;74(3):546-556.

20. Panea B, Ripoll G, Olleta JL, Sañudo C. Efecto del sexo y del cruzamiento sobre la calidad instrumental y sensorial y sobre la aceptación de la carne de añojos de la raza Avileña-negra ibérica. ITEA. 2011:107(3):239-250

21. Mahmood S, Basarab JA, Dixon WT, Bruce HL. Can potential for dark cutting be predicted by phenotype? Relationship between sex, carcass characteristics and the incidence of dark cutting beef. Can J Anim Sci 2016;(96):19-31.

22. McGilchrist P, Alston CL, Gardner GE, Thomson KL, Pethick DW. Beef carcasses with larger eye muscle areas, lower ossification scores and improved nutrition have a lower incidence of dark cutting. Meat Sci 2012;(92):474-480.

23. Sañudo C, Monsón F, Campo MM, Beltrán JA, Bello JM. Variación del pH en canales comerciales de cordero. En: Casasús-Pueyo I editor. XXXVII Jornadas de estudio y XI Jornadas sobre producción animal. Zaragoza, España 2005:703-705.

24. Hopkins DL, Stanley DF, Martin LC, Toohey ES, Gilmour AR. Genotype and age effects on sheep meat production. 3. Meat quality. Aus J Exp Agric 2007;47(10):11551164.

25. Vestergaard M, Oksbjerg N, Henckel P. Influence of feeding intensity, grazing and finishing feeding on muscle fibre characteristics and meat colour of semitendinosus, Longissimus dorsi and supraspinatus muscles of young bulls. Meat Sci 2000;54(2):177185.

26. Kadim IT, Mahgoub O, Al-Ajmi DS, Al-Maqbaly, RS, Al-Mugheiry SM, Bartolome DY. The influence of season on quality characteristics of hot-boned beef Longissimus thoracis. Meat Sci 2004;66(4):831-836.

27. Gregory NG. How climatic changes could affect meat quality. Food Research Int 2010;43(7):1866-1873. 
28. Chulayo AY, Bradley G, Muchenje V. Effects of transport distance, lairage time and stunning efficiency on cortisol, glucose, HSPA1A and how they relate with meat quality in cattle. Meat Sci 2016;(117):89-96.

29. Grandin T. Return sensibility problems after penetrating captive bolt stunning of cattle in commercial beef slaughter plants. J Am Vet Med Assoc 2002;(221):1258-1261.

30. Cadavez VAP, Xavier C, Gonzales-Barrona U. Classification of beef carcasses from Portugal using animal characteristics and $\mathrm{pH} /$ temperature decline descriptors. Meat Sci 2019;(153):94-102.

31. Jacob RH, Surridge VSM, Beatty DT, Gardner GE, Warner RD. Grain feeding increases core body temperature of beef cattle. Animal Prod Sci 2014;(54):444-449.

32. Warner RD, Thompson JM, Polkinghorne R, Gutzke D, Kearney GA. A consumer sensory study of the influence of rigor temperature on eating quality and ageing potential of beef striploin and rump. Animal Prod Sci 2014;(54):396-406.

33. Hargreaves A, Barrales L, Peña I, Larraín R, Zamorano L. Factores que influyen en el $\mathrm{pH}$ último e incidencia de corte oscuro en canales de bovinos. Cien Inv Agr 2004;31(3):155-166.

34. Lonergan HE, Zhang W, Lonergan SM. Biochemistry of postmortem muscle - lessons on mechanisms of meat tenderization. Meat Sci 2010;86(1):184-195.

35. Daniel WW, Cross CL. Biostatistics: A foundation for analysis in the health sciences. Tenth edition. New York, NY, USA; 2013.

36. Hahn GL. Dynamic responses of cattle to thermal heat loads. J Anim Sci 1999;77(2):10-20.

37. FAO. Manual de buenas prácticas para la industria de la carne. Organización de las Naciones Unidas para la Agricultura y la Alimentación. Roma, Italia; 2007.

38. HSA. Aturdimiento de animales por perno cautivo. Humane Slaughter Association. Wheat hampstead, Herts. AL4 8AN, UK; 2016.

39. Konica-Minolta. Precise color communication. Konica Minolta Sensing Inc. Japan. 2007.https://www.konicaminolta.com/instruments/knowledge/color/pdf/color_comm unication.pdf. Accessed 21 Aug, 2019.

40. Pérez-Linares C, Barreras A, Sánchez E, Herrera B, Figueroa-Saavedra F. The effect of changing the pre-slaughter handling on bovine cattle DFD meat. Rev MVZ Córdoba. 2015;20(3):4688-4697. 
41. Lattin J, Green PE, Carroll D. Analyzing multivariate data. Pacific Grove, CA, USA. Brooks/Cole Editor; 2003.

42. Hosmer DW, Lemeshow S. Applied logistic regression. 2nd ed. New York, NY, USA. Wiley-Interscience publication; 2000.

43. SAS Inc. Base SAS ${ }^{\circledR} 9.4$ Procedures Guide: Statistical procedures. 2nd ed. SAS Institute Inc. Cary, NC, USA: 2013.

44. Sánchez E, Navarro C, Sayas ME, Sendra E, Fernández J, Pérez JA. Análisis de diferentes factores que afectan la calidad de la carne: factores intrínsecos y ante mortem. En: Mota D, et al, editores. Bienestar animal. Una visión global en Iberoamérica. 3a ed. Barcelona, España: Elsevier; 2016: 495-510.

45. Garcia LG, Nicholson KL, Hoffman TW, Lawrence TE, Hale DS, Griffin DB, et al. National Beef Quality Audit-2005: Survey of targeted cattle and carcass characteristics related to quality, quantity, and value of fed steers and heifers. J Anim Sci 2008;(86):3533- 3543.

46. Moore MC, Gray GD, Hale DS, Kerth CR, Griffin DB, Savell JW, et al. National beef quality audit-2011: In-plant survey of targeted carcass characteristics related to quality, quantity, value, and marketing of fed steers and heifers. J Anim Sci 2012;90(13):51435151.

47. Donkersgoed JV, Jewison G, Bygrove S, Gillis K, Malchow D, McLeod G. Canadian beef quality audit 1998-99. Can Vet J 2001;(42):121-126.

48. Beef Cattle Research Council. National beef quality audit 2010/11 plant carcass audit. Canadian Cattlemen's Association; 2013.

49. Tadich N, Gallo C. Echeverría R, Van Schaik G. Efecto del ayuno durante dos tiempos de confinamiento y de transporte terrestre sobre algunas variables sanguíneas indicadoras de estrés en novillos. Arch Med Vet 2003;35(2):171-185.

50. NOM-033-SAG/ZOO-2014. Norma Oficial Mexicana NOM-033-SAG/ZOO-2014, Métodos para dar muerte a los animales domésticos y silvestres. Diario Oficial de la Federación; 2015.

51. MINAGRI, Reglamento sobre estructura y funcionamiento de mataderos, establecimientos frigoríficos, cámaras frigoríficas y plantas de desposte y fija equipamiento mínimo para tales establecimientos. Diario Oficial. Ministerio de Agricultura de Chile; 2009. 
52. Amtmann VA, Gallo C, Van-Schaik G, Tadich N. Relaciones entre el manejo antemortem, variables sanguíneas indicadoras de estrés y $\mathrm{pH}$ de la canal en novillos. Arch Med Vet 2006;38(3):259-264.

53. Grandin T. Recommended animal handling guidelines \& audit guide: a systematic approach to animal welfare. American Meat Institute Foundation; 2013.

54. Miranda-de-la-Lama GC, Leyva-García IG, Barreras-Serrano A, Pérez-Linares C, Sánchez-López E, Figueroa-Saavedra F, et al. Assessment of cattle welfare at a commercial slaughter plant in the northwest of Mexico. Trop Anim Health Prod 2012;(44):497-504.

55. Méndez-Medina RD, de Aluja AS, Rubio-Lozano MS, Braña-Varela D. Bienestar animal para operarios en rastros de bovinos. $1^{\mathrm{a}}$ ed. DF, México. SAGARPACONACYT-COFUPRO; 2013.

56. Muñoz D, Strappini A, Gallo C. Animal welfare indicators to detect problems in the cattle stunning box. Arch Med Vet 2012;(44):297-302.

57. Velarde A, Gispert M, Diestre A, Manteca X. Effect of electrical stunning on meat and carcass quality in lambs. Meat Sci 2003;63(1):35-38.

58. Ríos-Rincón FG, Acosta-Sánchez DC. Sacrificio humanitario de ganado bovino e inocuidad de la carne. Nacameh 2008;2(2):106-123.

59. Warris PD. Ciencia de la carne. 1a ed. Zaragoza, España: Editorial Acribia; 2003. 